\title{
Avaliação da Inteligência Emocional: A Relação entre Medidas de Desempenho e de Autorrelato ${ }^{1}$
}

\author{
Ana Cristina Fontes Costa ${ }^{2}$ \\ Luísa Maria Soares Faria \\ Universidade do Porto
}

\begin{abstract}
RESUMO - Apresenta-se a adaptação e validação do Teste de Vocabulário Emocional (TVE) ao contexto português, uma medida de desempenho de inteligência emocional, explorando as suas qualidades psicométricas e a validade de critério e convergente com uma medida de autorrelato, o Questionário de Competência Emocional - Revisto (QCE-R). O TVE, com 35 itens, desenvolvido por Takšic, Herambasic e Velemir (2003) no contexto croata, mede a capacidade para compreender emoções. A amostra incluiu 682 alunos do $10 .^{\circ}$ ano, entre 14 e 21 anos $(\mathrm{M}=15,5$; DP = 0,77) de diferentes cursos científico-humanísticos. Genericamente, o TVE revelou boas qualidades psicométricas, tendo-se correlacionado positivamente com o rendimento académico e evidenciado maior capacidade para o predizer do que o QCE-R, com o qual não estabeleceu qualquer associação.
\end{abstract}

Palavras-chave: inteligência emocional, medida de autorrelato, medida de desempenho, TVE, ensino secundário

\section{Assessment of Emotional intelligence: The Relation of Ability-Based and Self- Report Measures}

\begin{abstract}
This study presents the adaptation and validation to the Portuguese context of the Vocabulary Emotions Test (VET), an ability measure of Emotional Intelligence, particularly exploring their psychometrics properties and the criterion and convergent validity with a self-report measure, the Emotional Skills and Competence Questionnaire - Reviewed (ESCQ-R). Consisting of 35 items, the VET was constructed in Croatian settings by Takšic, Herambasic and Velemir (2003), and was developed to measure the ability to understand emotions. This study involved $68210^{\text {th }}$ graders, with ages between 14 and 21 years $(\mathrm{M}=15.5 ; \mathrm{SD}=.77)$ from several courses. The VET revealed good psychometric properties, showed positive correlations with academic achievement and evidenced better predictive validity than the ESCQ-R, with which no association was found.
\end{abstract}

Keywords: emotional intelligence, ability-based measure, self-report measure, VET, secondary education

Nos últimos anos o conceito de Inteligência Emocional (IE) tem suscitado um interesse crescente. De facto, o reconhecimento do papel adaptativo e potenciador das emoções no comportamento e ação humanos contribuíram para o incremento do valor da IE na investigação psicológica. $\mathrm{O}$ entusiasmo acentuou-se ao surgirem indicadores de que a IE se revelava potencialmente adaptativa ao facilitar o bem-estar emocional e global do indivíduo (Spence, Oades, \& Caputi, 2004), bem como a sua capacidade para realizar com mestria e ter sucesso (Goleman, 1995).

O conceito de IE foi apresentado por Salovey e Mayer (1990) e, desde então, uma série de modelos compreensivos de IE, com perspetivas mais complementares do que contraditórias (Ciarrochi, Chan, \& Caputi, 2000), surgiram, ampliando a concetualização relacionada com o tema, modificando pressupostos existentes ou contextualizando a IE face a outros construtos psicológicos (Gonzaga \& Monteiro, 2011). As diferentes concetualizações que

1 Apoio: Fundação para a Ciência e Tecnologia (FCT) através de uma bolsa de doutoramento atribuída à primeira autora Ana Costa $(\mathrm{FRH} /$ BD/72596/2010) sob a supervisão de Luísa Faria na FPCEUP

2 Endereço para correspondência: Faculdade de Psicologia e de Ciências da Educação da Universidade do Porto, Rua Alfredo Allen, Porto, Portugal. CEP: 4200-135, E-mail: 1faria@fpce.up.pt surgiram organizaram-se, essencialmente, em dois modelos principais: modelos de capacidade, em que a IE é definida como um conjunto de capacidades cognitivas que permitem o funcionamento emocional (Mayer \& Salovey, 1997) e modelos mistos que, por sua vez, integram uma vasta gama de características de personalidade (Bar-On, 2001; Goleman, 1995; Petrides \& Furnham, 2001).

Os modelos de capacidade concetualizam a IE como uma forma de inteligência que integra a emoção (Mayer \& Salovey, 1997; Mayer, Salovey, \& Caruso, 2004). Neste caso, a IE compreende uma série de competências que incluem a perceção e a expressão emocional, a facilitação emocional do pensamento, a compreensão e a regulação emocional. Nesse modelo de IE acredita-se que a emoção possa ser facilitadora do pensamento e que é possível processar cognitivamente as emoções (Mayer \& Salovey, 1993; Salovey \& Mayer, 1990). Mais ainda, uma vez que a IE possui características similares a outros tipos de inteligência, deverá refletir um tipo de capacidade ou de aptidão, estar relacionada com outras capacidades e desenvolver-se com a idade e com a experiência (Mayer et al., 2004; Neisser et al., 1996).

Apesar de alguns modelos mistos de IE fazerem referência à noção de capacidade no processamento e uso de informação emocional, combinam estas capacidades com outros traços e características, como o otimismo e a 
motivação (Goleman, 1995). Alguns autores consideram que os modelos mistos são relevantes na medida em que reconhecem que a emoção é afetada por múltiplos aspetos da personalidade (Goldenberg, Matheson, \& Mantler, 2006). No entanto, o facto deste tipo de modelo não apresentar uma nova concetualização do funcionamento emocional, e não se relacionar em particular com o conceito de emoção ou de inteligência (Matthews, Roberts, \& Zeidner, 2004), conduziu à limitação do seu uso, favorecendo a difusão dos modelos de capacidade na investigação da IE, perspetiva também partilhada neste estudo.

\section{Avaliação da IE}

O desenvolvimento dos modelos teóricos da IE tem sido acompanhado pelo desenvolvimento de medidas que testem o construto (Mayer, Caruso, \& Salovey, 2000). Desde o surgimento da primeira medida que avaliava um aspeto da IE, neste caso, a perceção de emoções em estímulos visuais (Mayer, DiPaolo, \& Salovey, 1990), novos dispositivos têm vindo a surgir e diferenciam-se, principalmente, em dois aspetos: baseiam-se nos dois modelos teóricos de IE apresentados e usam diferentes formas para a testar, desde testes de desempenho, inventários de autorrelato e, até, em alguns casos, a observação (Ciarrochi, Chan, Caputi, \& Roberts, 2001).

Alguns autores defendem que, em última análise, é a medida usada para avaliar a IE que determina a natureza do modelo de IE avaliado e não o contrário (Matthews et al., 2004; Petrides \& Furnham, 2000). Em particular, a literatura revela que as medidas de desempenho parecem ser as mais adequadas quando a IE é concetualizada como um tipo de capacidade, enquanto que os instrumentos de autorrelato se adaptam melhor quando a IE é perspetivada como compreendendo uma série de traços ou atributos (Ciarrochi, Chan, Caputi et al., 2001; Mayer, 2001).

De facto, o debate sobre quais as medidas de IE mais apropriadas e válidas tem evoluído ao longo dos últimos anos, mas são essencialmente os problemas metodológicos das medidas (autorrelato vs. realização) que mais têm limitado o seu alcance e valor heurístico. Neste sentido, a literatura sobre a IE tem-se questionado sobre o facto das medidas de autorrelato poderem ou não providenciar uma avaliação precisa do construto. Efetivamente, uma das limitações metodológicas mais apontadas às escalas de autorrelato é o facto de apenas avaliarem as crenças que o indivíduo possui acerca da sua própria competência na área da IE (Ciarrochi, Chan, Caputi et al., 2001; Schutte et al., 1998). Assim, quando as medidas de autorrelato são usadas, pensa-se que apenas são avaliadas características não cognitivas ou de personalidade. Não obstante, a IE concetualizada como uma capacidade é, não raras vezes, avaliada através de medidas de autorrelato (Barchard \& Hakstian, 2004; Schutte et al., 1998).

Mais ainda, este tipo de medidas, à semelhança da avaliação de outros construtos psicológicos, inclui algumas fraquezas e limitações, como a falta de confiança na opinião do indivíduo, bem como a maior vulnerabilidade a fatores de desejabilidade social (Roberts, Zeidner, \& Matthews, 2001; Schutte et al., 1998). Ora, é comummente referido o facto de as medidas de autorrelato refletirem não o atual nível de inteligência no funcionamento do indivíduo, ou seja, o seu atual desempenho, mas antes o percebido por si, que se torna dependente do nível de autoconhecimento ou de insight do próprio sobre as suas competências e capacidade para as reportar (Ciarrochi, Chan, Caputi et al., 2001). Acresce ainda, que a literatura sugere que as medidas de autorrelato de IE parecem coincidir de forma considerável com medidas de personalidade ou de temperamento (Barchard \& Hakstian, 2004; Brackett \& Mayer, 2003; Davies, Stankov, \& Roberts, 1998).

Dadas as críticas e limitações destas medidas, vários autores defenderam o desenvolvimento de indicadores objetivos de IE, baseados na capacidade e no desempenho pessoais (Mayer, Caruso, \& Salovey, 1999), propondo que testes objetivos clássicos, de desempenho ou de realização, sejam os pilares das investigações sobre IE, não sendo suficiente avaliar as perceções individuais de competência. De facto, a criação de medidas de desempenho que cumpram critérios semelhantes aos instrumentos desenvolvidos para a avaliação clássica da inteligência, parece tornar-se essencial para a IE assumir o estatuto de inteligência (Mayer et al., 1999). Assim, são necessários mais estudos que explorem as questões da validade das medidas e, a par do uso de medidas de perceção de competências (autorrelato), desenvolver formas alternativas de avaliação, como testes objetivos clássicos ou de realização (Ciarrochi, Chan, \& Bajgar, 2001).

Efetivamente, parece existir evidência das vantagens em usar medidas de desempenho ou de realização, sendo que uma das mais valorizadas prende-se com o facto de este tipo de medida aceder diretamente ao nível de desempenho do indivíduo na tarefa, ao contrário das medidas de autorrelato (Ciarrochi, Chan, Caputi et al., 2001; Schutte et al., 1998). Assim, de forma semelhante às medidas de inteligência geral, os testes de IE, baseados no desempenho, apresentam aos indivíduos problemas que têm apenas uma resposta correta (Mayer, 2001; Mayer et al., 2004), e que pode ser avaliada segundo critérios objetivos e predeterminados (Ciarrochi, Chan, Caputi et al., 2001). No entanto, apesar das consideráveis vantagens que as medidas de desempenho apresentam, a literatura tem revelado algumas das suas limitações no campo da IE. De facto, tem-se demonstrado difícil determinar objetivamente respostas corretas para estímulos com conteúdos emocionais e, consequentemente, aplicar critérios exatos na pontuação atribuída às tarefas propostas (Roberts et al., 2001). Por sua vez, questões relacionadas com as qualidades psicométricas parecem limitar a investigação com este tipo de medida (Matthews et al., 2004). Mais ainda, o facto de estes instrumentos serem exigentes em termos de tempo e de recursos, dificulta a sua aplicação em vários contextos e tem custos acrescidos (Goldenberg, et al., 2006).

Não obstante o debate em torno da avaliação no campo da IE e da utilidade de medidas de desempenho versus medidas de autorrelato, a literatura desenvolvida até à data tem evidenciado a fraca ou ausente associação entre os dois tipos de medidas (e.g., Barchard \& Hakstian, 2004; Brackett \& Mayer, 2003; Ciarrochi, Deane, \& Anderson, 2002; Davies et al, 1998; Lopes, Salovey, \& Straus, 2003). De facto, estudos que exploraram a validade convergente entre medidas 
commumente estabelecidas na área como o MSCEIT (MayerSalovey-Caruso-Emotional Intelligence Test), uma medida de desempenho, e o EQ-i (Emotional Quotient Inventory) e o SREIT (Self-Report EI Test), ambas medidas de autorrelato, evidenciaram fracas correlações entre si (Brackett \& Mayer, 2003), ou, ainda, quando foi considerado um mesmo modelo teórico de base, como no caso do MSCEIT e do TMMS (Trait Meta-Mood Scale), verificou-se que os resultados indicaram ausência de correlação entre alguns dos fatores do TMMS e o MSCEIT (Lopes et al., 2003).

De facto, a ausente ou fraca associação entre os dois tipos de medidas de IE parece ser consistente com os resultados da avaliação da inteligência no geral, onde, por norma, as medidas de desempenho e de autorrelato de QI (Quociente de Inteligência) apresentam fracas correlações entre si (Furnham \& Rawles, 1999; Paulhus, Lysy, \& Yik, 1998). De facto, Sternberg (1988) acredita que esta fraca associação poderá ser devida ao facto de os atributos avaliados pelas duas medidas não serem correspondentes entre si. Mais ainda, outros autores consideraram que tal discordância entre as medidas poderia dever-se por um lado à sobrevalorização das perceções dos participantes nas medidas de autorrelato, devido a tendências narcisistas, ou pelo facto dos participantes basearem a sua concetualização de inteligência naquilo que pensam ser a definição de inteligência de quem os avalia (Dunning \& Cohen, 1992), e, por outro lado, à pouca capacidade de insight, interesse ou preocupação com este atributo (Campbell \& Lavalle, 1993). No entanto, e uma vez que os tipos de medidas de IE se baseiam não raras vezes nos mesmos modelos teóricos e que se propoêm atingir objetivos comuns, surge cada vez mais a preocupação por parte dos investigadores em explorar questões relacionadas com a validade convergente dos instrumentos. De facto, considerando as vantagens e limitações dos dois tipos de medidas, torna-se necessário utilizar métodos variados de avaliação, a partir dos quais se poderá contribuir para a promoção do estatuto científico da IE e para a validade das medidas (Roberts et al., 2001).

Efetivamente, este estudo, partilhando desta preocupação atual, visa contribuir para a clarificação de questões sobre a avaliação da IE, particularmente, apresentando um estudo de validação de uma medida de desempenho ou de realização de IE, o Teste de Vocabulário Emocional (TVE), e explorando as relações que este tipo de medida estabelece com uma medida de autorrelato de IE, o Questionário de Competência Emocional - Revisto (QCE-R).

Tendo por base uma concetualização da IE que a perspetiva num modelo de competência ou de capacidade, diferente dos ancorados nos atributos de personalidade ou fatores motivacionais do indivíduo (Brackett \& Mayer, 2003), estas medidas resultam do modelo definido por Mayer e Salovey (1997). Apesar de segundo este modelo a IE se operacionalizar de forma unitária, esta pode ser divisível em quatro categorias diferentes (Mayer, Salovey, \& Caruso, 2000). A primeira categoria diz respeito à identificação e perceção emocional, e envolve o reconhecimento e a entrada da informação no sistema emocional. A segunda e a terceira categorias, facilitação emocional do pensamento e compreensão emocional, respetivamente, envolvem o processamento posterior de informação e enfatizam a resolução de problemas. Globalmente, a facilitação emocional do pensamento (segunda categoria) envolve o uso da emoção para melhorar o processamento cognitivo, enquanto que a categoria de compreensão emocional (terceira categoria) envolve o processamento cognitivo da emoção. O quarto nível, gestão emocional, diz respeito à autorregulação emocional e à regulação das emoções nas outras pessoas (Mohoric, Takšic, \& Duran, 2010). Nesse sentido, a IE é um conjunto de capacidades interrelacionadas que permitem perceber, reconhecer e gerar emoções de modo a apoiar o pensamento, compreender sentimentos e cognições subsequentes e regular as emoções de modo reflexivo, de forma a promover o desenvolvimento emocional e intelectual dos indivíduos (Mayer \& Salovey, 1997). O presente estudo, para além de apresentar a adaptação e validação de uma medida de desempenho de IE - Teste de Vocabulário Emocional (TVE) -, ao explorar as relações que este tipo de medida de desempenho estabelece com uma medida de autorrelato de IE - Questionário de Competência Emocional - Revisto (QCE-R) -, também pretende contribuir para aprofundar o debate sobre o diálogo entre os dois tipos de medidas e para fomentar a clarificação de questões de validade das medidas de IE.

\section{Método}

\section{Participantes}

Participaram neste estudo 682 alunos do $10 .^{\circ}$ ano do ensino secundário da zona do Grande Porto, com idades compreendidas entre os 14 e os 21 anos $(\mathrm{M}=15,5$; DP $=$ $0,77)$. A maior parte dos alunos é do sexo feminino $(52,9 \%)$, do nível sócio-profissional (NSP) e cultural (NSC) médioalto $(41,6 \%$ e $32,7 \%$ respetivamente) e do curso científicohumanístico de Ciências e Tecnologias $(45,7 \%)$.

\section{Instrumentos}

Teste de Vocabulário Emocional (TVE). O Vocabulary Emotions Test é uma medida de capacidade ou desempenho de IE originalmente desenvolvida por Takšic, Herambasic e Velemir (2003), no contexto educativo croata com alunos do ensino secundário, a partir do modelo de Mayer e Salovey (1997), baseando-se, particularmente, na capacidade para Compreender a Emoção (terceira categoria). O TVE compreende 35 itens, que correspondem a palavras alvo, com conotação emocional, com múltiplas opções de resposta (escolha do sinónimo que mais se aproxima da palavra alvo). Cada palavra alvo tem uma opção de resposta correta, que é cotada com 1 ponto, podendo o resultado final do TVE ser de 0 a 35 pontos possíveis, a que correspondem, respetivamente, a ausência e a totalidade de respostas corretas no teste.

Genericamente, a versão original do teste apresenta boas qualidades psicométricas: correlações moderadas com outros testes de Inteligência (California Tests of Mental Maturity - Subteste de Vocabulário $-r=0,67, p=0,00$ e Subteste de Pensamento Lógico $-r=0,33, p=0,00)$ e 
Inteligência Emocional (Analysis of Emotions Test $-r$ $=0,46, p=0,00)$ e explica $44 \%$ de variância única sobre o subteste de Vocabulário do California Tests of Mental Maturity. Apresenta também um nível de consistência interna elevado $(\alpha=0,90)$ e demonstra, no que diz respeito à média da variância e da correlação interitens, valores moderados (0,22; Takšic \& Mohoric, 2008).

A adaptação portuguesa do TVE (Costa, Faria, \& Takšic, 2011) foi, globalmente, consistente com os resultados encontrados na versão original. O estudo dos vários indicadores de sensibilidade para o TVE revelou que: i) os valores da média $(18,9)$ e mediana $(20)$ são próximos; ii) os coeficientes de assimetria $(-0,58)$ e de curtose $(0,11)$ são inferiores à unidade; e iii) os valores mínimo (3) e máximo (30) encontram-se afastados assegurando a dispersão dos resultados, sendo possível inferir sobre a boa sensibilidade do instrumento. No que se refere à análise das propriedades dos itens que constituem o TVE, quer o índice de dificuldade $(\mathrm{M}=0,55 ; \mathrm{DP}=0,22)$, quer o poder discriminativo dos itens $(\mathrm{M}=0,27$; $\mathrm{DP}=0,12)$, demonstraram ser aceitáveis. O TVE apresentou ainda bons indicadores de consistência interna, com resultados de alfa de Cronbach de 0,77 , sendo que estes valores se encontram abaixo dos encontrados na amostra original $(0,90)$. Por outro lado, os valores médios de covariância e de correlação interitens variaram entre 0,02 e 0,10 , respetivamente, sendo também inferiores ao valor de 0,22 encontrado no estudo original do instrumento.

Questionário de Competência Emocional - Revisto (QCE-R). O QCE-R é uma medida de autorrelato de IE, com três dimensões (Perceção Emocional, Expressão Emocional e Capacidade para Lidar com a Emoção) e 42 itens, traduzida e adaptada para o contexto português por Lima Santos e Faria (2005) e revista em 2011 pelos mesmos autores (Faria \& Lima Santos, 2011). Tem por base a concetualização teórica da IE de Mayer e Salovey (1997), e resultou da adaptação ao contexto português do Emotional Skills and Competence Questionnaire, que foi originalmente desenvolvido na Croácia por Takšic (2000), tendo sido utilizado em contexto académico e laboral, em várias culturas (Faria et al., 2006), apresentando, globalmente, boas qualidades psicométricas. De facto, extrai três fatores concordantes com as dimensões teóricas, revela alfas entre 0,72 e 0,82 , correlações positivas entre as suas dimensões (entre 0,49 e 0,54 ) e um ajustamento local e global aceitáveis com valores de saturação dos itens entre os 0,55 e 0,78 e valores R2 entre 0,31 e 0,60, bem como valores de $\mathrm{NNFI}=0,93, \mathrm{CFI}=0,94$ e $\mathrm{RMSEA}=0,04$ (Stocker \& Faria, 2012).

Instrumentos de autoconceito e autoeficácia. Para estudar a validade de critério do TVE foram também administrados o Self-Description Questionnaire III (SDQIII), adaptado por Faria e Fontaine (1992), que inclui três dimensões (Autoconceito Verbal, Matemático e Assuntos Escolares), num total de 30 itens, e a Escala de Autoeficácia Académica (EAEA), desenvolvida por Pina Neves e Faria (2006) também constituída por três dimensões (Autoeficácia a Português, Matemática e Escolar Geral) com 22 itens. Esses instrumentos foram amplamente usados no contexto académico português, tendo demonstrado boas qualidades psicométricas (e.g. Faria \& Fontaine, 1992; Pina Neves \& Faria, 2006).
Para recolher dados sobre as características sociodemográficas da amostra foi desenvolvido, de raíz para este estudo, o Questionário Sociodemográfico e Académico (QSDA). Esse questionário, com questões de resposta fechada, com 14 itens, integra questões sobre informações sociodemográficas dos participantes, como seja, idade, género, nível socioprofissional e cultural, bem como questões relativas aos seus dados académicos, como, por exemplo, o ano de escolaridade ou a área científica frequentada pelo aluno.

No sentido de obter o nível de rendimento académico dos alunos foram recolhidas, nas escolas, as classificações dos alunos relativas ao último período do ano letivo ( $3 .^{\circ}$ período). De facto, considerou-se que as classificações dos alunos no 3 . $^{\circ}$ e último período seriam as mais representativas do nível atingido pelo aluno durante o ano letivo.

\section{Procedimento}

Para este estudo foram selecionadas oito escolas com ensino secundário de diferentes zonas geográficas da cidade do Porto. Após a autorização para a realização do estudo por parte da escola procedeu-se à seleção aleatória das turmas e, de acordo com a disponibilidade das escolas, à marcação dos dias e horários de administração dos instrumentos. Foi ainda pedido o consentimento informado dos Encarregados de Educação dos alunos para a autorização da participação no estudo.

O TVE foi administrado em conjunto com o QCE-R, SDQIII, EAEA e QSDA, em administrações coletivas com turmas inteiras, em horários letivos, sempre com a presença na sala do respetivo professor da turma e de um investigador. Em cada administração foi explicado o objetivo do estudo e garantida a confidencialidade dos dados. A média de preenchimento dos questionários foi de 30 minutos por turma.

\section{Resultados}

Considerando a análise da sensibilidade do TVE, os resultados indicaram que a amostra de estudantes do ensino secundário obteve resultados globais no TVE entre 4 e 32 pontos, tendo sido o resultado de 25 pontos o mais frequente. $\mathrm{O}$ estudo dos vários indicadores de sensibilidade revelou que, ao nível das medidas de tendência central, de distribuição e de dispersão, os resultados são satisfatórios. A análise da sensibilidade do TVE permitiu concluir que: i) os valores de média $(22,4)$ e mediana $(23,0)$ são próximos; ii) os coeficientes de assimetria $(-0,66)$ e de curtose $(0,65)$ são inferiores à unidade; e iii) os valores mínimo (4) e máximo (32) encontram-se afastados, assegurando a dispersão dos resultados. No que se refere à análise das propriedades dos itens que constituem o TVE, particularmente os índices de dificuldade dos itens, os valores médios encontrados foram de $0,65(\mathrm{DP}=0,24)$, tendo sido 0,75 , o índice de dificuldade dos itens mais frequente (oito itens com este valor). Verificou-se que existem dois itens do teste que apresentam um índice de dificuldade no extremo inferior da escala, abaixo dos 0,15 , e, por outro lado, três itens apresentaram valores que se situam 
no extremo superior da escala, com um índice de dificuldade de 0,95 . No que concerne à análise do poder discriminativo dos itens do TVE, o valor médio registado para esta amostra foi de $0,19(\mathrm{DP}=0,09)$.

Relativamente aos indicadores de consistência interna, o instrumento demonstrou, globalmente, boa consistência interna, com resultados de alfa de Cronbach de 0,71 para a amostra total. No que se refere ao género, na subamostra dos alunos verificaram-se valores de alfa de 0,71 , sendo que para a subamostra das alunas os valores de consistência interna foram muito semelhantes, isto é, de 0,72.
Ainda no âmbito da validade de critério foi explorada a capacidade de predição das duas medidas de IE sobre o rendimento académico dos alunos. De facto, apesar de ambas as medidas de desempenho e de autorrelato terem demonstrado capacidade de predizer o rendimento académico dos alunos, foi o TVE que apresentou maior capacidade para prever a média global das notas dos alunos, bem como a nota de Português e Matemática, enquanto que o QCE-R não revelou capacidade de predizer a nota de Matemática dos alunos (cf. Tabela 1). Nos casos em que a capacidade de predição foi significativa, ambas as medidas verificaram

Tabela 1. Validade de critério do TVE e do QCE-R em relação ao rendimento académico

\begin{tabular}{ccccccc}
\hline \multirow{2}{*}{ Variáveis } & \multicolumn{2}{c}{ Média das Notas } & \multicolumn{2}{c}{ Nota a Português } & \multicolumn{2}{c}{ Nota a Matemática } \\
\cline { 2 - 6 } & $\mathbf{R 2}$ & $\boldsymbol{\Delta R 2}$ & $\mathbf{R 2}$ & $\boldsymbol{\Delta R 2}$ & $\mathbf{R 2}$ & $\Delta \mathbf{R 2}$ \\
\hline TVE & $0,126^{*}$ & $0,124^{*}$ & $0,122^{*}$ & $0.121^{*}$ & $0,098^{*}$ & $0,096^{*}$ \\
QCE-R & $0,020^{*}$ & $0,018^{*}$ & $0,007^{* *}$ & $0,005^{* *}$ & 0,007 & 0,005 \\
EE & $0,010^{* *}$ & $0,008^{* *}$ & 0,003 & 0,001 & 0,007 & 0,005 \\
PE & $0,009^{* *}$ & $0,008^{* *}$ & $0,009^{* *}$ & $0,008^{* *}$ & 0,006 & 0,004 \\
Cap. & $0,008^{* *}$ & $0,007^{* *}$ & 0,003 & 0,001 & 0,001 & $-0,001$ \\
\hline
\end{tabular}

Nota: EE - Expressão Emocional; PE - Perceção Emocional; Cap.- Capacidade para Lidar com a Emoção.

${ }^{*} p<0,01 .{ }^{* *} p<0,05$

No sentido de verificar a validade de critério do TVE com o rendimento académico, este demonstrou correlacionar-se positiva e significativamente com o rendimento académico dos alunos, em particular com a nota de Português $(r=0,36$; $p<0,01)$ e com a média global das notas dos alunos $(r=0,37$; $p<0,01)$, no $3^{\circ}$ e último período escolar. Verificou-se que o instrumento apresentou uma maior associação com a média global das notas e com a nota a Português, do que com a nota de Matemática $(r=0,33 ; p<0,01)$ no último período escolar.

Tabela 2. Correlações entre o TVE e as dimensões do Autoconceito e Autoeficácia

\begin{tabular}{lc}
\hline \multicolumn{1}{c}{ Dimensões } & TVE \\
\hline AC Verbal & $0,33^{* *}$ \\
AC Assuntos Escolares & $0,27 * *$ \\
AC Matemática & $0,16^{* *}$ \\
AE Português & $0,23^{* *}$ \\
AE Geral & $0,22^{* *}$ \\
AE Matemática & $0,21 * *$ \\
\hline
\end{tabular}

uma maior capacidade de predição para a média global das notas dos alunos, seguida da nota de Português e Matemática.

Por outro lado, no sentido de verificar a validade convergente com outros construtos relacionados, o TVE revelou associar-se às várias dimensões do autoconceito e da autoeficácia dos alunos. Em particular revelou estar correlacionado mais fortemente com as dimensões verbais do SDQIII $(r=0,33 ; p<0,01)$ e EAEA $(r=0,23 ; p<0,01)$, do que com as dimensões relacionadas com Matemática e assuntos académicos gerais (cf. Tabela 1).

Finalmente, e no sentido de explorar a validade convergente entre as duas medidas de IE, foi avaliado o seu grau de associação, não se tendo verificado quaisquer associações significativas entre a medida de desempenho e de autorrelato de IE analisadas. De facto, o TVE não demonstrou correlacionar-se com a escala total de QCE-R nem com nenhuma das respetivas dimensões (cf. Tabela 3 ). No que diz respeito às associações encontradas, apenas as dimensões do QCE-R se associaram fortemente entre si e com a escala total.

Tabela 3. Correlações entre o TVE e o QCE-R e as suas subdimensões.

\begin{tabular}{ccccccccc} 
& TVE & $\boldsymbol{p}$ & QCE-R & $\boldsymbol{p}$ & EE & $\boldsymbol{p}$ & PE & $\boldsymbol{p}$ \\
\hline QCE-R & 0,06 & 0,12 & & & & & & \\
EE & 0,05 & 0,23 & $0,83^{* *}$ & 0,00 & & & \\
PE & 0,06 & 0,13 & $0,79 * *$ & 0,00 & $0,44^{* *}$ & 0,00 & $0,45^{* *}$ & 0,00 \\
Cap. & 0,03 & 0,45 & $0,79 * *$ & 0,00 & $0,50 * *$ & 0,00 & 00 \\
\hline
\end{tabular}

Nota: EE - Expressão Emocional; PE - Perceção Emocional; Cap.- Capacidade para Lidar com a Emoção; p= nível de significância. $* * \mathrm{p}<0,01$. 


\section{Discussão}

Este estudo apresentou evidências de que o TVE é uma medida de desempenho consistente e válida na avaliação da IE e, ainda, apresentou resultados adicionais quanto à validade de critério e convergente do TVE com uma medida de autorrelato de IE. Genericamente, as análises demonstraram que os resultados totais do TVE seguem uma distribuição normal, com valores de média e mediana próximos, coeficientes de assimetria e de curtose inferiores à unidade e valores mínimos e máximos afastados, evidenciando boa sensibilidade. Uma análise mais particular dos itens do TVE revelou que os índices de dificuldade dos itens $(M=0,65 ; D P=0,24)$ se aproximaram do valor 0,50 recomendado (Anastasi \& Urbina, 2000), e que os resultados médios obtidos são semelhantes aos encontrados no estudo original do instrumento $(\mathrm{M}=0,62 ; \mathrm{DP}=0,21)$ e um pouco inferiores aos do estudo piloto da adaptação portuguesa do instrumento $(\mathrm{M}=0,55 ; \mathrm{DP}=0,22)$. No entanto, apesar de os valores médios serem satisfatórios, alguns itens apresentam índices de dificuldade extremados na escala, e deste modo, mais afastados do índice recomendado, podendo estar a ser excessivamente fáceis para alunos de ensino secundário, ou, por outro lado, excessivamente difíceis. No que se refere à análise do poder discriminativo dos itens do TVE, o valor médio encontrado $(M=0,19 ; \mathrm{DP}=0,09)$ foi considerado razoável. Para a maioria dos objetivos de testagem, são preferíveis os itens mais próximos do valor de dificuldade 0,50, tal como foi referido. Desse modo, os níveis dos índices de discriminação dos itens que favorecem este nível de dificuldade são mais apropriados para a seleção dos itens (Anastasi \& Urbina, 2000). A diferença verificada entre os valores dos índices de dificuldade e poder discriminativo dos itens da versão original e adaptada do TVE poderá deverse aos procedimentos de adaptação linguística e cultural do instrumento. Neste sentido, os itens que se afastam consideravelmente dos valores recomendados precisam de ser melhorados ou redefinidos para se tornarem mais equilibrados na sua dificuldade e poder discriminativo.

A consistência interna da versão portuguesa do TVE foi globalmente boa, com resultados de alfa de Cronbach de 0,71 para a amostra total. No entanto, os valores obtidos encontram-se abaixo dos encontrados na amostra original $(0,90)$, bem como do estudo piloto da adaptação portuguesa do instrumento $(0,77)$.

Ao explorar a validade de critério do instrumento, o TVE demonstrou estabelecer uma associação positiva e moderada com o rendimento académico dos alunos, sendo particularmente mais intensa a relação evidenciada com a média global das notas dos alunos, no último período escolar, seguido das suas notas de Português. De facto, o papel da IE em contexto académico é muitas vezes retratado na literatura pela associação positiva que estabelece com o rendimento académico geral (Barchard, 2003; Brackett \& Mayer, 2003). Do mesmo modo, os resultados evidenciaram a capacidade das medidas de desempenho e de autorrelato predizerem o rendimento académico, apesar de, estes resultados não serem consistentes na literatura (Brackett \& Mayer, 2003; Brackett, Mayer, \& Warner, 2004; O’Connor \& Little, 2003). De facto, o contexto académico parece limitar a capacidade de explicação da IE, por comparação com os contextos organizacionais, uma vez que alguns autores consideram que as exigências no contexto escolar são menos variadas, o que pode reduzir a variabilidade da capacidade de predição da IE neste contexto particular (Van Rooy \& Viswesvaran, 2004). Por outro lado, e de acordo com Barchard (2003), verificouse a superioridade da medida de desempenho para predizer o rendimento académico. De facto, concetualmente parece lógico que uma medida de capacidade de IE, que se baseia em competências cognitivas como o raciocínio, possa ser um preditor mais eficaz do rendimento académico (O'Connor \& Little, 2003), medido, nesse caso, pela média global das notas dos alunos.

Mais ainda, o TVE demonstrou maior capacidade para predizer a média global das notas dos alunos e a sua nota a Português do que a nota de Matemática. Como foi anteriormente referido, sendo este um teste de vocabulário emocional, focalizado na compreensão emocional com base em palavras com conotação emocional, a avaliação deste indicador é baseada na dimensão verbal e nas competências linguísticas, parecendo favorecer desta forma a maior relação que o instrumento estabelece com a nota de Português.

Tal como era expectável, das várias dimensões de autoconceito e autoeficácia dos alunos foram as dimensões verbais aquelas que mais fortes associações estabeleceram com o TVE. O facto deste instrumento ser uma medida de capacidade focalizada essencialmente na compreensão emocional e de a tarefa apresentada para a avaliação deste indicador ser do tipo verbal, parece ter conduzido à maior associação verificada.

Embora fundadas num mesmo enquadramento teórico, neste estudo as duas medidas de IE (desempenho vs. autorrelato) não se correlacionaram entre si. De facto, a ausente ou a fraca associação entre estes tipos de medidas, é congruente com estudos prévios sobre a convergência das medidas de desempenho e de autorrelato de IE (Barchard \& Hakstian, 2004; Brackett \& Mayer, 2003; Davies et al, 1998). De facto, também no contexto da inteligência geral, não raras vezes o relato dos indivíduos acerca das suas competências está apenas fracamente correlacionado com medidas de desempenho de QI (Mayer et al., 2000; Mayer et al., 2004; Paulhus et al., 1998). Alguns autores acreditam que esta menor associação poderá ficar a dever-se ao facto dos dois tipos de medidas, apesar de terem objetivos de avaliação comuns, não conseguirem ser convergentes na sua forma de avaliar as competências tendo como consequência não avaliarem os mesmos atributos (Sternberg, 1988).

Por outro lado, apesar de ambas as medidas de IE partilharem o mesmo modelo teórico e os mesmos objetivos de avaliação, o tipo de medida, mais do que o modelo teórico em que se baseia a mesma, pode, em última instância, estar a determinar a natureza do modelo de IE que está a ser testado (Matthews et al., 2004; Petrides \& Furnham, 2000). Em particular, sendo a IE perspetivada como um conjunto de capacidades intercorrelacionadas, então as medidas de desempenho ou realização partilham de um conjunto de características mais adequadas para avaliar este tipo de competências, enquanto os instrumentos de autorrelato estão dirigidos a um número de traços ou atributos não cognitivos (Ciarrochi, Chan, Caputi et al., 2001; Mayer, 2001), possivelmente justificando a não convergência das medidas. 
Mais ainda, o facto de os scores nas medidas de autorrelato serem em média mais elevados quando comparados com os das medidas de desempenho, sugere que os indivíduos possam sobrestimar as suas competências emocionais (Goldenberg et al., 2006), quer devido à pouca capacidade de insight das suas reais competências nestas matérias (Campbell \& Lavalle, 1993), quer devido à tentativa de se valorizar de acordo com o que acreditam ser socialmente valorizado (Dunning $\&$ Cohen, 1992). Deste modo, apesar de se propor avaliar um modelo de capacidade, o QCE-R enquanto medida de autorrelato poderá estar constrangido por algum viés e por limitações próprias de outras medidas de autorrelato de IE, conduzindo à dificuldade em encontrar convergência com a medida de desempenho. De facto, apesar de este estudo não ter verificado a associação entre os dois tipos de medidas, é notório que concetualmente ambas partilham pontos comuns. Neste sentido, é necessário que se clarifiquem as limitações, principalmente a nível da operacionalização das medidas de autorrelato, bem como a especificidade das medidas de desempenho, por forma a desmistificar o tipo de diálogo estabelecido entre ambas.

O presente estudo, além de ter procurado contribuir para a clarificação de questões de validade das medidas de avaliação de IE, ao demonstrar que o Teste de Vocabulário Emocional apresentou potencialidades de utilização no contexto educativo português, por ter revelado qualidades psicométricas satisfatórias e evidências de validade enquanto medida de desempenho de IE, contribuiu também para o debate sobre a convergência e diálogo estabelecidos entre medidas de desempenho e de autorrelato de IE. A investigação futura, no entanto, não exclui a necessidade de replicação de estudos de validade das várias medidas com amostras mais alargadas e em outros contextos de existência, devendo centrar-se essencialmente no desenvolvimento de medidas mais refinadas de inteligência emocional.

\section{Referências}

Anastasi, A., \& Urbina, S. (2000). Testagem psicológica. Porto Alegre: Artmed Editora.

Barchard, K. A. (2003). Does emotional intelligence assist in the prediction of academic success? Educational and Psychological Measurement, 63, 840-858. doi: 10.1177/0013164403251333

Barchard, K. A., \& Hakstian, A. R. (2004). The nature and measurement of emotional intelligence abilities: Basic dimensions and their relationships with other cognitive ability and personality variables. Educational and Psychological Measurement, 64, 437-462. doi:10.1177/0013164403261762

Bar-On, R. (2001). Emotional intelligence and self-actualization. In J. Ciarrochi, J. P. Forgas, \& J. D. Mayer (Eds.), Emotional intelligence in everyday life: A scientific inquiry (pp. 82-97). Philadelphia: Taylor \& Francis.

Brackett, M. A., \& Mayer, J. D. (2003). Convergent, discriminant and incremental validity of competing measures of emotional intelligence. Personality and Social Psychology Bulletin, 29, 1147-1158. doi: 10.1177/0146167203254596
Brackett, M. A., Mayer, J. D., \& Warner, R. M. (2004). Emotional intelligence and its relation to everyday behaviour. Personality and Individual Differences, 36, 1387-1402. doi:10.1016/ S0191-8869(03)00236-8

Campbell, J. D., \& Lavalle, L. F. (1993). Who I am? The role of self-concept confusion in understanding the behaviour of people with low self-esteem. In R.F. Baumeister (Ed.), Selfesteem: The puzzle of low self-regard (pp. 3-20). New York: Plenum Books.

Ciarrochi, J., Chan, A. Y. C., \& Caputi, P. (2000). A critical evaluation of the emotional intelligence construct. Personality and Individual Differences, 28, 539-561. doi: 10.1016/S01918869(99)00119-1

Ciarrochi, J., Chan, A. Y. C., \& Bajgar, J. (2001). Measuring emotional intelligence in adolescents. Personality and Individual Differences, 31, 1105-1119. doi: http://dx.doi. org/10.1016/S0191-8869(00)00207-5

Ciarrochi, J., Chan, A., Caputi, P., \& Roberts, R. (2001). Measuring emotional intelligence. In J. Ciarrochi, J. P. Forgas, \& J. D. Mayer (Eds.), Emotional intelligence in everyday life: A scientific inquiry (pp. 25-45). Philadelphia: Taylor \& Francis.

Ciarrochi, J., Deane, F. P., \& Anderson, S. (2002). Emotional intelligence moderates the relation between stress and mental health. Personality and Individual Differences, 32, 197-209.

Costa, A., Faria, L., \& Takšic, V. (2011). Vocabulary of emotions test (VET): A cross-cultural validation in Portuguese and Croatian contexts. Psychological Topics, 21(3), 413-424.

Davies, M., Stankov, L., \& Roberts, R. D. (1998). Emotional intelligence: In search of an elusive construct. Journal of Personality and Social Psychology, 75, 989-1015. doi:10.1037/0022-3514.75.4.989

Dunning, D., \& Cohen, G. L. (1992). Egocentric definitions of traits and abilities in social judgment. Journal of Personality and Social Psychology, 63, 341-355.

Faria, L., \& Fontaine, A. M. (1992). Estudo de adaptação do Self-Description Questionnaire III (SDQ III) a estudantes universitários portugueses. Psychologica, 8, 41-49.

Faria, L., \& Lima Santos, N. (2011). Questionário de Competência Emocional (QCE). In C. Machado, M. M. Gonçalves, L. S. Almeida \& M. R. Simões (Eds.), Instrumentos e contextos de avaliação psicológica (vol. I, pp. 67-80). Coimbra: Almedina.

Faria, L., Lima Santos, N., Taksic, V., Räty, H., Molander, B., Holmström, S., . . Toyota, H. (2006). Cross-cultural validation of the Emotional Skills and Competence Questionnaire (ESCQ). Psicologia, 2, 95-127.

Furnham, A., \& Rawles, R. (1999). Correlations between selfestimated and psychometrically measured IQ. The Journal of Social Psychology, 139(4), 405-410.

Goldenberg, I., Matheson, K., \& Mantler, J. (2006). The assessment of emotional intelligence: A comparison of performancebased and self-report methodologies. Journal of Personality Assessment, 86, 33-45. doi: 10.1207/s15327752jpa8601_05

Goleman, D. (1995). Inteligência emocional. Lisboa: Temas e Debates.

Gonzaga, A. R., \& Monteiro, J. K. (2011). Inteligência emocional no Brasil: um panorama da pesquisa científica. Psicologia: Teoria e Pesquisa, 27(2), 225-232.doi:http://dx.doi.org/10.1590/ S0102-37722011000200013 
Lima Santos, N., \& Faria, L. (2005). Inteligência emocional: Adaptação do "Emotional Skills and Competence Questionnaire" (ESCQ) ao contexto português. Revista da Faculdade de Ciências Humanas e Sociais da UFP, 2, 275-289.

Lopes, P. N., Salovey, P., \& Straus, R. (2003). Emotional intelligence, personality, and the perceived quality of social relationships. Personality and Individual Differences, 35, 641-658.

Mayer, J. D. (2001). A field guide to emotional intelligence. In J. Ciarrochi, J. P. Forgas, \& J. D. Mayer (Eds.), Emotional intelligence in everyday life (pp. 3-24). Philadelphia: Taylor $\&$ Francis.

Mayer, J. D., Caruso, D., \& Salovey, P. (1999). Emotional intelligence meets traditional standards for an intelligence. Intelligence, 27, 267-298. doi: 10.1016/S0160-2896(99)000161

Mayer, J. D., Caruso, D. R., \& Salovey, P. (2000). Selecting a measure of emotional intelligence: The case for ability scales. In R. Bar-On \& J. D. A. Parker (Eds.), Handbook of emotional intelligence: Theory, development, assessment, and application at home, school, and in the workplace (pp. 320-342). San Francisco: Jossey-Bass.

Mayer, J. D., DiPaolo, M. T., \& Salovey, P. (1990). Perceiving affective content in ambiguous visual stimuli: A component of emotional intelligence. Journal of Personality Assessment, 54, 772-781.

Mayer, J. D., \& Salovey, P. (1993). The intelligence of emotional intelligence. Intelligence, 17, 433-442. doi: 10.1016/01602896(93)90010-3

Mayer, J. D., \& Salovey, P. (1997). What is emotional intelligence? In P. Salovey \& D. J. Sluyter (Eds.), Emotional development and emotional intelligence. Educational implications (pp. 3-31). New York, NY: Basic Books.

Mayer, J. D., Salovey, P., \& Caruso, D. (2000). Emotional intelligence as Zeitgeist, as personality, and as a mental ability. In R. Bar-On \& J. D. A. Parker (Eds.), Handbook of emotional intelligence: Theory, development, assessment, and application at home, school, and in the workplace (pp. 92-117). San Francisco: Jossey-Bass.

Mayer, J. D., Salovey, P., \& Caruso, D. (2004). Emotional intelligence: Theory, findings and implications. Psychological Inquiry, 15, 197-215. doi: 10.1207/s15327965pli1503_02

Matthews, G., Roberts, R. D., \& Zeidner, M. (2004). Seven myths about emotional intelligence. Psychological Inquiry, 15, 179196.

Mohoric, T., Takšic, V., \& Duran, M. (2010). In search of "the correct answer" in an ability-based emotional intelligence (EI) test. Studia Psychologica, 52, 219-228.

Neisser, U., Boodoo, G., Bourchard, T. J., Jr., Boykin, A. W., Brody, N., Ceci, S. J., . . . Urbina, S. (1996). Intelligence: Knowns and unknowns. American Psychologist, 51, 77-101. doi: 10.1037/0003-066X.51.2.77

O’Connor, R. M. Jr, \& Little, I. S. (2003). Revisiting the predictive validity of emotional intelligence: Self-report versus abilitybased measures. Personality and Individual Differences, 35, 1893-1902. doi:10.1016/S0191-8869(03)00038-2

Paulhus, D. L., Lysy, D. C., \& Yik, M. S. M. (1998). Self-report measures of intelligence: Are they useful as proxy IQ tests? Journal of Personality, 66,525-554. doi: 10.1111/14676494.00023
Petrides, K. V., \& Furnham, A. (2000). On the dimensional structure of emotional intelligence. Personality and Individual Differences, 29, 313-320. doi: 10.1016/S0191-8869(99)001956

Petrides, K. V., \& Furnham, A. (2001). Trait emotional intelligence: Psychometric investigation with reference to established trait taxonomies. European Journal of Personality 15, 425-448. doi: 10.1002/per.416

Pina Neves, S., \& Faria, L. (2006). Construção, adaptação e validação da Escala de Auto-Eficácia Académica (EAEA). Psicologia, $X X(2), 45-68$.

Roberts, R. D., Zeidner, M., \& Matthews, G. (2001). Does emotional intelligence meet traditional standards for an intelligence? Some new data and conclusions. Emotion, 1, 196-231. doi: 10.1037//1528-3542.1.3.196

Salovey, P., \& Mayer, J. D. (1990). Emotional Intelligence. Imagination, Cognition and Personality, 9, 185-211.

Schutte, N. S., Malouff, J. M., Hall, L. E., Haggerty, D. J., Cooper, J. T., Golden, C. J., \& Dornheim, L. (1998). Development and validation of a measure of emotional intelligence. Personality and Individual Differences, 25, 167-177. doi:10.1016/S01918869(98)00001-4

Spence, G., Oades, L. G., \& Caputi, P. (2004). Trait emotional intelligence and goal self-integration: important predictors of emotional well-being? Personality and Individual Differences, 37, 449-461. doi:10.1016/j.paid.2003.09.001

Sternberg, R. J. (1988). The triarchic mind: A new theory of human intelligence. New York: Penguin Books.

Stocker, J., \& Faria, L. (2012). Competência percebida no ensino secundário: Do conceito à avaliação através de um questionário compósito. Psicologia, 26(2), 113-140.

Takšic, V. (2000). Emotional Skills and Competence Questionnaire (ESCQ). Rijeka: Author's edition.

Takšic, V., Herambasic, D., \& Velemir, B. (2003). Verbal Emotional Test (VET 35). Rijeka: Authors' edition.

Takšic, V., \& Mohoric, T. (2008, julho). Vocabulary of Emotion Test (VET): Ability measure of emotional intelligence. Comunicação apresentada no XXIX Congresso Internacional de Psicologia, Berlim/Alemanha.

Van Rooy, D. L., \& Viswesvaran, C. (2004). Emotional Intelligence: A Meta-Analytic Investigation of predictive Validity and Nomological Net. Journal of Vocational Behavior, 65, 71-95.

Recebido em 19.03.2012

Primeira decisão editorial em 16.09.2013

Versão final em 08.11.2013

Aceito em 20.12.2013 